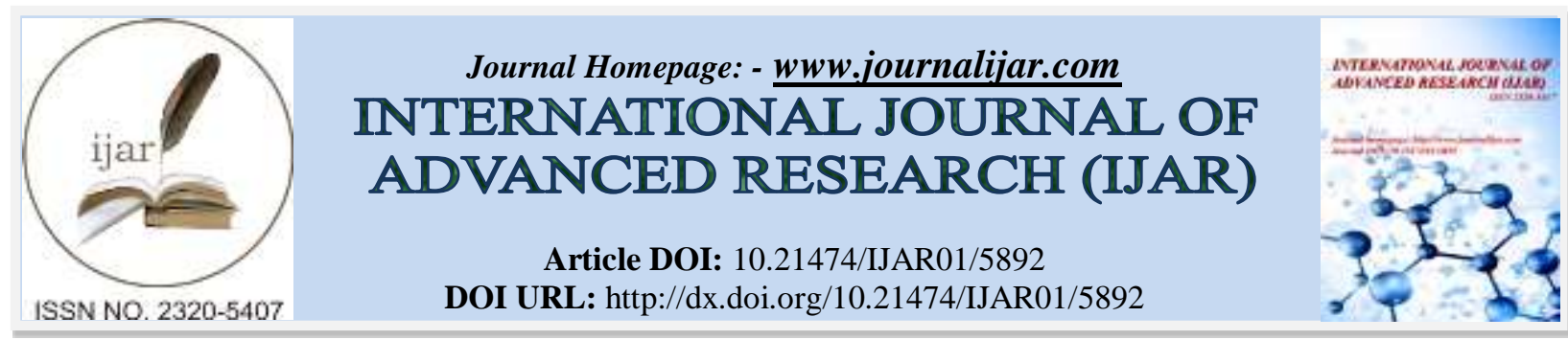

RESEARCH ARTICLE

\title{
A COMPARATIVE ANALYSIS OF ANTIFUNGAL SUSCEPTIBILITY OF KERATITIS ASSOCIATED FUNGAL BIOFILM AND PLANKTONIC CULTURES.
}

\author{
Jayangshu Sengupta. MS ${ }^{1}$, Suman Saha. PhD ${ }^{1}$, Debdulal Banerjee. PhD $^{2}$ and Santi M Mandal. PhD ${ }^{3}$. \\ 1. Cornea and Refractive Services, Priyamvada Birla Aravind Eye Hospital, Kolkata, India. \\ 2. Dept of Botany and Forestry, Vidyasagar University, Midnapore, India. \\ 3. Central Research Facility, Indian institute of Technology, Kharagpur, India.
}

\section{Manuscript Info}

Manuscript History

Received: 17 September 2017

Final Accepted: 19 October 2017

Published: November 2017

Key words:-

Fungal biofilm, Keratitis, Antifungal

Susceptibility.

\begin{abstract}
Biofilm are considered as the most important developmental characteristics in ocular infections. Biofilm eradication is a major challenge today to overcome the incidence of drug resistance and treatment failures in cases of fungal keratitis. This report demonstrates the in vitro ability of biofilm formation by three common keratitisassociated fungal pathogens, namely, Aspergillus fumigatus, Fusarium solani, and Candida albicans. Antifungal sensitivity testing performed for both planktonic cells and biofilm revealed the sessile phenotype to be resistant at MIC levels for the planktonic cells and also at higher concentrations. Antifungal susceptibility testing following resuspension of the sessile form into a planktonic mode demonstrated an intermediate MIC value with development of resistance compared to the original planktonic state. This demonstrates that antifungal resistance acquired by biofilms is permanent and transferred to subsequent generations thereby converting a sensitive phenotype into a resistant form.
\end{abstract}

Copy Right, IJAR, 2017,. All rights reserved.

\section{Introduction:-}

Corneal blindness is a significant health problem across the world. According to the World Health Organization report, it is estimated that ocular trauma and corneal ulceration result in 1.5 to 2 million new patients of corneal blindness annually, posing a major public health concern for developing countries like India (Whitcher et al., 2001).

Worldwide, fungi account for around 50\% cases of infective keratitis. Srinivasan et al from South India reported that $44 \%$ of all central corneal ulcers were caused by fungi (Srinivasan, 1997). Molds are most common in tropical parts of the world like India and southeastern United States with more than 70 species identified as responsible pathogens. Involvement of new species is also reported regularly. In India, according to previous reports, Aspergillus sp. and Fusarium sp. are the commonest organisms followed by dematiaceous fungi along with an increasing incidence of Candida keratitis (Sengupta et al., 2012).On the other hand, in temperate regions like northern parts of America, Candida albicans forms the most common isolate although Fusarium keratitis is increasingly recognized (Sun et al.,2007). 
Recent advances have provided evidence that microbial adherence to the human biotic surface constitutes the first step towards development of a biofilm community which is contemplated to be responsible for more than $65 \%$ of human infections (Donlan, 2002). A biofilm is a structured microbial community of cells enclosed in a matrix of extracellular polymeric substances (EPS), growing on a substrate, and displaying phenotypic features that vary from their planktonic or free-floating counterparts (Costerton,1995). Such an existence presumably allows the microbial cells to survive in hostile environments, enhances their resistance to physical and chemical pressures, and promotes metabolic cooperation. Although bacterial biofilms have been extensively studied since the mid 1980s, little attention was paid to medically relevant fungal biofilms until the past decade. Much of our understanding about fungal biofilm formation has been gained through the studies of biofilms formed by Candida species on artificial surfaces (Kumamoto et al., 2002) such as stents, shunts, prostheses, implants, endotracheal tubes, pacemakers, and various types of catheters, to name a few (Shimuzu et al., 2000; Ramage et al., 2005).

The role of fungal biofilms in ophthalmic setting, particularly with reference to infective keratitis, is unknown. However, this may possess a potentially important role in pathogenesis of the disease condition as well as outcome, as biofilm formation tends to induce an antifungal resistance and alter the response to therapy. While our knowledge is restricted to fungal biofilm formation on implanted medical devices and its role in invasive infections, recently, few reports have speculated a similar pathologic mechanism in contact lens related fungal keratitis. In vitro studies have been conducted to demonstrate the formation of fungal biofilm on a contact lens substrate and have also shown a high degree of antifungal resistance (Imamura et al., 2008; Sengupta et al, 2012).

In this experimental study we have studied three fungal strains-Aspergillus fumigatus, Fusarium solani, and Candida albicans - isolated from patients with infective keratitis to compare the antifungal susceptibility between planktonic culture, biofilm mode of growth and post-biofilm dispersed planktonic culture(BDPC).

\section{Materials and Methods:-}

The fungal pathogens - Aspergillus sp, Fusarium sp, and Candida sp - isolated from cases of fungal keratitis attending the Cornea Services of Priyamvada Birla Aravind Eye Hospital, Kolkata were selected for this study. The three strains have been characterized earlier based on their genetic data. (Saha et al., 2009). Briefly, the DNA was isolated from the culture medium and the quality evaluated on 1.2\% agarose gel. Species confirmation was done using D1/D2 region of LSU (Large SubUnit: 28SrDNA) based on PCR technique. Ribosomal ITS (internal transcribed spacer) region is amplified by using primers 5'-GGTTGGTTTCTTTTCCT-3' and 5'AAGTAAAAGTCGTAACAAGG-3'.

The fungal species were collected from mature solid medium culture plate (Sabouraud dextrose agar) and mixed with liquid RPMI 1640 (Himedia, India) and incubated for $24 \mathrm{hrs}$ at $30^{\circ} \mathrm{C}$ to obtain the relevant turbidity of $0.5 \times 10^{4}$ $\mathrm{CFU} / \mathrm{ml}$. The selected isolates were submerged in 96 wells, flat bottom polystyrene plates containing $2 \mathrm{ml}$ of RPMI 1640 medium, inoculated with respective fungal strain as a inoculum dose of $3.5 \times 10^{6} \mathrm{CFU} / \mathrm{ml}$ and incubated for 72 hours at $30^{\circ} \mathrm{C}$. After 72 hours the each well was washed with $1 \times$ PBS repeatedly to remove the non-biofilm cells. Biofilm formation was confirmed under fluorescence microscopy using Concavalin A-Alexa Fluor 488 (Invitrogen, Carlsbad, CA, USA) conjugate (ConA) $(2001 \mathrm{~g} / \mathrm{ml})$ counterstaining and XTT (2,3-bis(2-methoxy-4-nitro-5-sulfophenyl-2H-tetrazolium-5-carboxanilide; Sigma) reduction assay using a Multiskan Spectrum-1500 spectrophotometer (Thermo Scientific) as described previously (Sengupta et al,,2012).

The following antifungals were used for determination of antifungal susceptibility namely liposomal amphotericin $\mathrm{B}$, voriconazole, ketoconazole, itraconazole, natamycin, and anidulafungin. Antifungal agents were prepared as previously described (Anil et al., 2002). Antifungal drug sensitivity tests were performed in accordance with national committee for clinical laboratory standards (NCCLS) guidelines. The following antifungal discs (Hi Media, India) were used which included voriconazole, natamycin, amphotericin B, fluconazole, ketoconazole and anidulafungin. The selective stains were inoculated into SDA media, cultured for 2-5 days and the inoculum was diluted to $0.5 \times 104 \mathrm{CFU} / \mathrm{ml}$ with saline water. The inoculum $(0.5 \mathrm{ml})$ were plated in SDA media, dried for $15 \mathrm{~min}$ at $37^{\circ} \mathrm{C}$ then antifungal discs were placed on the media surface by following disc diffusion methods. The plates were incubated at $30^{\circ} \mathrm{C}$ and the zone diameters were observed for each strain after $48 \mathrm{hrs}$ incubation. The zone diameters were measured at which colonies were remaining normal. The zone diameters were interpreted as proposed by the manufacturer's guidelines. The minimum inhibitory concentration of selective antifungal (voriconazole, amphotericin B, ketoconazole, itraconazole and anidulafungin) (Hi Media, India) were determined according to the Antifungal Susceptibility Testing Subcommittee of the European Committee on Antibiotic Susceptibility Testing 
(AFST-EUCAST) method (Cuenca-Estrella et al., 2003) using RPMI 1640 supplemented with $2 \%$ glucose as the test medium, and an inoculum size of $0.5 \times 10^{5}$ to $2.51 \times 10^{5}$ cells $/ \mathrm{ml}$.

The minimum biofilm eradication concentration (MBEC) of the selected antifungals were determined as by Melo et al. The respective antifungal agents were added to the biofilm in serial double-diluted concentrations ( 0.5 to 500 $\mu \mathrm{g} / \mathrm{ml}$ ) and total volume of $250 \mu 1$ with RPMI medium and solutions of each antifungal agent was also prepared in RPMI medium directly, incubated further for 24 hours at $35^{\circ} \mathrm{C}$. A series of antifungal agent-free wells and biofilmfree wells was also included to serve as positive and negative controls, respectively. After incubation, the remaining biofilm metabolic activity was quantified by the XTT-reduction assay. Testing of these isolates were performed in triplicate.

To evaluate MIC for biofilm derived planktonic cells, biofilms was formed as mentioned above. After 24 hrs and 48 hrs, the biofilms were washed gently and transferred to $2 \mathrm{ml}$ micro centrifuge tube for vortexing for $5 \mathrm{~min}$. Serial dilutions of antifungals were made and transferred to the 96 well plate. The votexed RPMI liquid medium was added to each 96 well plate with $10 \mu 1$ of antifungal. These biofilm derived planktonic cells (BDPC) were incubated with all the tested antifungal either for 24 or $48 \mathrm{hrs,} \mathrm{and} \mathrm{MIC} \mathrm{was} \mathrm{determined} \mathrm{as} \mathrm{described} \mathrm{earlier.}$

\section{Results:-}

\section{Biofilm formation and quantification:-}

Fluorescence microscopy demonstrated biofilm formation by all three studied strains that were quantified with the XTT-reduction assay. Mature biofilm formation was observed after 72-hrs incubation for all strains with Candida forming the highest amount of biofilm followed by Aspergillus and Fusarium.

\section{Antifungal sensitivity for planktonic state.}

All the three strains were sensitive to most of the antifungals tested by disc diffusion except ketoconazole, which showed intermediate activity against Fusarium (Table I). Estimation of MIC values showed that Voriconazole was most effective antifungal against Aspergillus, amphotericin B was most effective in Fusarium whereas ketoconazole and itraconazole showed lowest MIC against Candida sp (Table 2).

Mean biofilm eradication concentration and antifungal sensitivity of post biofilm dispersed planktonic state:At biofilm state mean biofilm eradication concentration was measured and found ineffective upto $64 \mu \mathrm{g} / \mathrm{ml}$ for all selected antifungals against the isolated fungal species. Antifungal susceptibility test was also performed after biofilm breakdown where the MIC's showed an intermediate value, lower compared to biofilm state but not attaining the values in the planktonic state, (Table 3 ) for all the tested antifungals.

\section{Discussion:-}

Antifungal sensitivity tests have developed greatly in the recent years; various methods such as agar dilution, disc diffusion, concentration gradient and liquid medium dilution are applied in various laboratories in India. We used disc diffusion methods following NCCLS guidelines (2002) where the test mediators, inoculum concentration, incubation temperature for different mycelial fungus are clearly defined. This study showed that in planktonic form of growth, Aspergillus fumigatus and Fusarium solani were more sensitive to voriconazole compared to other tested azoles, natamycin and anidulafungin. while Amphotericin B was effective against Candida as well as showed good sensitivity against both Aspergillus and Fusarium. However, due to poor penetration in cornea and requirement of high dosage to ensure the adequate concentration in eye, it is not preferred as first line of management in cases of fungal keratitis (Chang et al, 2006). Natamycin is the standard of care in many countries especially in developing countries where natamycin was effective in superficial infection and not in severe cases. Lalitha et al. reported that voriconazole had a lowest MIC against Aspergillus while fluconazole and miconazole showed resistance against maximum number of species. Ketoconazole and itaconazole showed invitro sensitivity against Candida rather than other filamentary fungi (Lalitha et al, 2007). However, as showed in this study that resistance of Fusarium and Aspergillus to azoles had increased greatly. Simultaneously, it is important to remember that all the results of antifungal sensitivity tests in vitro are completely not coincident with the therapeutic effects in vivo as the immune defence of hosts also plays important roles in the occurrence of fungal infection. If the pathogen was sensitive to an antifungal treatment with this medication will be effective up to $90 \%$ of the patients and in case of resistance therapy 
it will be effective in $60 \%$ of patients [15]. One of the factors that may play a role in such discordance may be due to existence of invivo biofilm community in these recalcitrant cases of infective keratitis.

One of the defining characteristics of biofilms is their increased resistance to antimicrobial agents. Fungi have been reported to be up to 1000-fold more resistant to antifungal agents than planktonic free floating cells, yet this recalcitrance to antimicrobial therapy has yet to be fully elucidated (Ramage et al., 2011,Xu et al., 2000). Antifungal resistance is both complex and multi-factorial. It can be inducible in response to a compound, or an irreversible genetic change resulting from prolonged exposure. Specifically, these include alterations or overexpression of target molecules, active extrusion through efflux pumps, limited diffusion, tolerance and cell density, which are all characterized mechanisms utilized by fungi to combat the effects of antifungal treatment (Jabra Rizk et al., 2004).

Planktonic cells generally rely on irreversible genetic changes to maintain a resistant phenotype, whereas biofilms are able to persist due to their physical presence and the density of the population, which provides a resistant phenotype irrespective of defined genetic alterations (Kuhn et al., 2002; Kuhn et al., 2004). The results of our study demonstrate that all the studied organisms were sensitive to the tested antifungals in planktonic mode while high degree of resistance was acquired when they attained biofilm state. Interestingly, this study showed that the post biofilm dispersed planktonic cultures were also resistant to the tested antifungals which implies a permanent genetic alteration incurred during biofilm formation which is responsible for converting a non resistant pathogenic strain to a resistant phenotype.

\section{Conclusion:-}

To conclude, biofilm formation by Aspergillus, Fusarium and Candida isolated from cases of infective keratitis confers antifungal resistance which is permanent and persists even after breakdown of the biofilm, probably explaining that such resistance is acquired due to a combination of factors which include both morphological and genetic changes. This may be one of the key factors responsible for failure of antifungal therapy in majority of cases of infective keratitis. Further studies need to be performed to identify the genetic changes responsible for generation of resistant fungal phenotypes.

TABLE I

\begin{tabular}{|l|c|c|c|c|c|c|}
\hline Fungi & Ampho B & Natamycin & Ketoconazole & Itraconazole & Anidulafungin & Voriconazole \\
\hline A. fumigatus & Sensitive & Sensitive & Sensitive & Sensitive & Sensitive & Sensitive \\
\hline C. albicans & Sensitive & Sensitive & Sensitive & Sensitive & Sensitive & Sensitive \\
\hline $\boldsymbol{F}$. solani & Sensitive & Sensitive & Intermediate & Sensitive & Intermediate & Sensitive \\
\hline
\end{tabular}

Antifungal Susceptibility Testing By Disc Diffusion

TABLE II

\begin{tabular}{|l|c|c|c|c|c|c|c|c|c|c|}
\hline Organism & \multicolumn{2}{|l|}{ Ampho B } & \multicolumn{2}{l|}{ Voriconazole } & \multicolumn{2}{l|}{ Itraconazole } & \multicolumn{2}{l|}{ Ketoconazole } & \multicolumn{2}{l|}{ Anidulafungin } \\
\hline & Disc & Broth & Disc & Broth & Disc & Broth & Disc & Broth & Disc & Broth \\
\hline A.fumigatus & 2 & 2.5 & 0.6 & 0.12 & 2 & 1.5 & 4 & 2.5 & 1 & 1 \\
\hline F.solani & 1 & 1 & 4 & 8 & 8 & 12 & 8 & 8 & 8 & 12 \\
\hline C.albicans & 2 & 3 & 16 & 16 & 0.32 & 0.2 & 0.6 & 1.2 & 1 & 2 \\
\hline
\end{tabular}

Comparative data of antifungal susceptibility (mic) using disc diffusion and microbroth dilution technique. All measurements are in $\mu \mathrm{g} / \mathrm{ml}$

TABLE III

\begin{tabular}{|l|c|c|c|c|c|c|c|c|c|c|}
\hline Organism & \multicolumn{2}{|l|}{ Ampho B } & \multicolumn{2}{l|}{ Voriconazole } & \multicolumn{2}{l|}{ Itraconazole } & \multicolumn{2}{l|}{ Ketoconazole } & \multicolumn{2}{l|}{ Anidulafungin } \\
\hline & MBEC & BDPC & MBEC & BDPC & MBEC & BDPC & MBEC & BDPC & MBEC & BDPC \\
\hline A.fumigatus & $>64$ & 16 & $>64$ & 8 & $>64$ & 32 & $>64$ & 16 & $>64$ & 24 \\
\hline F.solani & $>64$ & 12 & $>64$ & 24 & $>64$ & 32 & $>64$ & 24 & $>64$ & $>64$ \\
\hline C.albicans & $>64$ & 24 & $>64$ & 32 & $>64$ & 12 & $>64$ & 24 & $>64$ & 8 \\
\hline
\end{tabular}

Comparative data of mean biofilm eradication concentration (mbec) and biofilm dispersed planktonic cell (bdpc) mic values. All measurements are in $\mu \mathrm{g} / \mathrm{ml}$ 


\section{Reference:-}

1. Anil S, Ellepola AN, Samaranayake LP. The impact of polyene, azole, and DNA analogue antimycotics on the cell surface hydrophobicity of Candida albicans and Candida tropicalis in HIV infection. Mycopathologia. 2002; 153: 179-185.

2. Chang DC, Grant GB, O'Donnell K, Wannemuehler KA, Noble-Wang J, Rao CY, et al. Multistate outbreak of Fusarium keratitis associated with use of a contact lens solution. JAMA. 2006; 296: 953-63.

3. Costerton JW, Lewandowski Z, Caldwell DE, Korber DR, Lappin-Scott HM. Microbial biofilms. Annu Rev Microbiol. 1995; 49:711-745.

4. Cuenca-Estrella M, Moore CB, Barchiesi F, Bille J, Chryssanthou E, Denning DW, Donnelly J P, Dromer F, Dupont B, Rex J H, Richardson M D, Sancak B, Verweij P E, Rodríguez-Tudela J L, the AFST Subcommittee of the European Committee on Antimicrobial Susceptibility Testing. Multicenter evaluation of the reproducibility of the proposed antifungal susceptibility testing method for fermentative yeasts of the Antifungal Susceptibility Testing Subcommittee of the European Committee on Antimicrobial Susceptibility Testing (AFST-EUCAST). Clinical Microbiology and Infection. 2003; 9: 467-474.

5. Donlan RM, Costerton JW. Biofilms: survival mechanisms of clinically relevant microorganisms. Clin Microbiol Rev 2002; 15:167-93.

6. Imamura Y, Chandra J, Mukherjee PK, Lattif AA, Szczotka-Flynn LB, Pearlman E, Lass JH, O'Donnell K, Ghannoum MA.. Fusarium and Candida albicans biofilms on soft contact lenses: model development, influence of lens type, and susceptibility to lens care solutions. Antimicrob Agents Chemother. 2008; 52:171182.

7. Jabra-Rizk MA, Falkler WA, Meiller TF. Fungal biofilms and drug resistance. Emerg Infect Dis. 2004;10:1419.

8. Kuhn DM, George T, Chandra J, Mukherjee P K, Ghannoum MA Antifungal Susceptibility of Candida Biofilms: Unique Efficacy of Amphotericin B Lipid Formulations and Echinocandins Antimicrobial Agents Chemothery.2002; 46:1773-1780.

9. Kuhn DM, Ghannoum MA. Candida biofilms: antifungal resistance and emerging therapeutic options Curr Opin Investig Drugs. 2004; 5:186-197.

10. Kumamoto CA. Candida biofilms. Curr Opin Microbiol. 2002; 5:608-611.

11. Lalitha P, Shapiro BL, Srinivasan M, Prajna NV, Acharya NR, et al.Antimicrobial susceptibility of Fusarium, Aspergillus, and otherfilamentous fungi isolated from keratitis. Arch Ophthalmol (2007) 125: 789-793.

12. Mowat E, Lang S, Williams C, McCulloch E, Jones B, Ramage G. Phase-dependent antifungal activity against Aspergillus fumigatus developing multicellular filamentous biofilms. J. Antimicrob. Chemother.2008; 62: 1281-1284.

13. Ramage G, Rajendran R, Gutierrez-Correa M, Jones B, Williams C. Aspergillus biofilms: clinical and industrial significance. FEMS Microbiology letter.2011; 332:89-97.

14. Ramage G, Rajendran R, Sherry L, Williams C. Fungal Biofilm Resistance. International Journal of Microbiology.2012.

15. Saha S, Banerjee D, Khetan A, Sengupta J. Epidemiological profile of fungal keratitis in urban population of West Bengal, India. Oman J Ophthalmol. 2009; 3:114-118.

16. Sengupta J, Khetan A, Saha S, Banerjee D, Gangopadhyay N, Pal D. Candida Keratitis: emerging problem in India. Cornea. 2012; 31:371-5.

17. Sengupta J, Saha S, Khetan A, Sarkar SK, Mandal SM. Effects of lactoferricin B against keratitis-associated fungal biofilms. J Infect Chemother. $2012 ; 18(5): 698-703$.

18. Shimizu K, Kobayakawa S, Tsuji A, Tochikubo T. Biofilm formation on hydrophilic intraocular lens material Curr Eye Res. 2006; 31:989-997.

19. Srinivasan M, Gonzales CA, George C, et al. Epidemiology and aetiological diagnosis of corneal ulceration in Madurai, South India. British Journal of Ophthalmology 1997; 81:965-971.

20. Sun RL, Jones DB, Wilhelmus KR. Clinical characteristics and outcome of Candida keratitis. Am J Ophthalmol. 2007; 143:1043-1045.

21. Whitcher, JP.,Srinivasan. M.,Upadhyay, M. P. Corneal blindness: a global perspective. Bulletin of the World Health Organization. 2001; 79:214-221.

22. Xu KD, McFeters GA, Stewart PS. Biofilm resistance to antimicrobial agent Microbiology.2000; 146 :547-549. 